\title{
Fuentes proteínicas no tradicionales y su efecto sobre el crecimiento y supervivencia, durante la masculinización del pez Cichlasoma urophthalmus (Perciformes: Cichlidae)
}

\author{
Carlos A. Cuenca-Soria*, Leonardo I. Navarro Angulo, Alfonso Castillo-Domínguez, Carolina E. Melgar Valdes, \\ Xchel A. Pérez-Palafox, Mateo Ortiz-Hernández
}

Universidad Juárez Autónoma de Tabasco. División Académica Multidisciplinaria de los Ríos. Carretera Tenosique-Estapilla km 1, 86901, Tenosique, Tabasco, México; cccp0900@hotmail.com, leonardo.navarro@ujat.mx, alfonsin75@gmail.com, cemv81@gmail.com, xapp39@gmail. com, mateo121@hotmail.com; * Autor de correspondencia

Recibido 12-IV-2016 • Corregido 25-V-2016 • Aceptado 03-VII-2016

\begin{abstract}
Nontraditional protein sources and their effect on growth and survival, during masculinization, of the fish Cichlasoma urophthalmus (Perciformes: Cichlidae). In Southeastern Mexico, aquaculture has been seriously affected by the high costs of commercial food. As part of the search for alternative foods we tested the freshwater fish Astyanax aeneus and the snail Pomacea flagellata as possible protein sources for Cichlasoma urophthalmus. We applied fluoxymesterone androgenic hormone for 45 days. One kilogram of experimental food (P. flagellate/A. aeneus) and one of commercial food were sprayed with solution of fluoxymesterone/absolute ethanol, at $5 \mathrm{mg} / \mathrm{L} / \mathrm{kg}$ rate (hormone/alcohol/food). The experimental and artificial foods (THA and SHC, respectively) were tested in fry C. urophthalmus, synchronously with their control groups (TA and SC, respectively), by triplicate. THA produced the highest specific growth and final mean weight $(p<0.05)$. There were differences in sex ratios but considering the dosage they are not relevant.
\end{abstract}

Key words: Sources, protein, nontraditional, Cichlasoma, urophthalmus.
RESUMEN: En el sureste de México, la acuicultura se ha visto seriamente afectada por los altos costos de los alimentos comerciales. Como parte de la búsqueda de alimentos no tradicionales, probamos el pez de agua dulce Astyanax aeneus y el caracol Pomacea flagellata como posibles fuentes de proteínas para Cichlasoma urophthalmus. Aplicamos la hormona androgénica fluoximesterona durante 45 días. Un kilogramo de dieta experimental (P. flagellate/A. aeneus) y uno de los alimentos comerciales se rociaron con la solución de fluoximesterona/etanol absoluto, en $5 \mathrm{mg} / \mathrm{L} / \mathrm{tasa} \mathrm{kg}$ (hormona/alcohol/alimento). La dieta experimental y el alimento comercial (THA y SHC, respectivamente) fueron probados en crías de $C$. urophthalmus, sincrónicamente con sus grupos de control (TA y SC, respectivamente), por triplicado. THA produjo el mayor crecimiento específico y la media final peso $(p<0,05)$. No hubo diferencias en la proporción de sexos $(p>0,05)$, teniendo en cuenta que la dosis utilizada no fue relevante.

Palabras clave: Fuentes, proteínicas, no convencionales, Cichlasoma, urophthalmus.
El costo principal en el alimento del pez, sigue siendo la fuente de proteína. Se han realizado una serie de esfuerzos en los últimos años para identificar fuentes alternativas de proteína adecuadas para el crecimiento de organismos acuáticos diversos. Los alimentos balanceados para especies acuícolas de importancia comercial, tiene una demanda creciente en el mundo, lo cual ha generado un interés en la búsqueda de nuevos ingredientes no convencionales para camarón (Casas et al., 2006); tilapia y trucha (El Sayed, Martínez \& Moyano, 2000; Lee et al., 2002). Así mismo, la disponibilidad de fuentes proteínicas no convencionales para uso acuícola, en zonas del trópico-húmedo del sureste mexicano es vasta, sobresaliendo los crustáceos, moluscos, peces, subproductos de la actividad agropecuaria, actualmente poco estudiadas y explotadas, sobre todo debido al escaso valor comercial que tienen en éstas regiones, como muestran los trabajos de Frías-Quintana et al. (2010) y Cuenca et al. (2013a). Dentro de las fuentes de proteína piscícolas no convencionales, sobresalen la sardinita $A$. aeneus, que solo es utilizada como cebo para la pesca artesanal de otras especies de importancia comercial, debido a 
su pequeña talla. El género Astyanax, es uno de los más ampliamente distribuidos no solo en México, sino en el continente americano (D’artola, Arias \& Páramo, 2008).

Los caracoles del género Pomacea, cuyo aprovechamiento como fuente alimenticia en México, figura el caracol tote $P$. flagellata, comúnmente colectado en las riberas de los cuerpos de agua del sureste mexicano. A pesar de la pérdida constante de su hábitat, presenta altos porcentajes de eclosión, múltiples desoves mensuales, desarrollo directo y corto período de incubación, rápido crecimiento y amplio rango de tolerancia a condiciones ambientales, lo hacen un excelente candidato para su explotación acuícola (Iriarte-Rodríguez \& Mendoza-Carranza, 2007). Paralelamente, las especies piscícolas nativas constituyen importantes pesquerías, generando fuentes de empleo y de alimentos para la población (Perera-García et al., 2008). Dentro de las investigaciones relacionadas con las especies nativas, sobresale el manual del cultivo de la mojarra latinoamericana, $C$. urophthalmus de Martínez-Palacios y Ross (1994), donde se abordan aspectos de cultivo de la especie. El trabajo de Uscanga-Martínez et al. (2012), lleva acabo una evaluación del requerimiento óptimo de proteína en juveniles de mojarra tenhuayaca Petenia splendida, hallándose que tal nivel resultó ser alrededor del 45\%. Siguiendo con esta especie, Treviño et al. (2011) estudiaron los cambios morfológicos y funcionales, para dilucidar la diferenciación del tracto digestivo, en larvas de $P$. splendida. Cuenca et al. (2013 b) evaluaron la actividad enzimática digestiva en juveniles de C. urophthalmus, revelando un amplio espectro enzimático, propio de un pez omnívoro; mientras que Frías-Quintana et al. (2010), probaron dietas experimentales en larvas del pejelagarto Atractosteus tropicus, a base de ingredientes proteínicos no convencionales, como harina de calamar, de jaiba, de sangre de res, de pollo y cerdo, encontrándose buena digestibilidad, crecimiento y supervivencia, con dietas a base de éstos dos últimos ingredientes.

Por otro lado, el cíclido maya es una especie euritópica, ya que se localiza en ríos, lagos, lagunas, ambientes salobres (lagunas costeras, ciénegas), manifestaciones cársticas (cenotes, aguadas, cavernas), ambientes con influencia marina (petenes, manglares y pastos marinos) son biotopos comunes de este pez, (Chávez-Sánchez et al., 2000). Con base en sus hábitos alimenticios, la especie ha sido considerada como omnívora, (Chávez-Lomelí et al., 1989). Su dieta se compone, principalmente, de materia orgánica, crustáceos, camarones, anfípodos, moluscos, isópodos, poliquetos, huevos de invertebrados y restos vegetales (Caso-Chávez et al., 1986; MartínezPalacios \& Ross, 1988). Por su alta capacidad de adaptación al cautiverio, el cíclido Maya C. urophthalmus, ha sido una de las cíclidos nativos hasta ahora más estudiados, con miras a consolidar su cultivo (Pérez-Sánchez \& Páramo-Delgadillo, 2008; Cuenca et al., 2013b).

Ante el encarecimiento de la harina de pescado, es menester buscar nuevas fuentes alternativas de proteína, para el diseño de alimentos inertes para especies nativas, con amplio potencial acuícola. Nuestro objetivo fue evaluar el efecto de la inclusión de harina de sardinita $A$. aeneus y caracol tote $P$. flagellata en la dieta, sobre el crecimiento y sobrevivencia de crías de C. urophthalmus, durante su masculinización.

\section{MÉTODOS}

Las larvas de C. urophthalmus fueron obtenidas a partir del lote de reproductores (3:1, hembras-macho), mantenidos en las instalaciones del área de Acuacultura, de la División Académica Multidisciplinaria de los Ríos (DAMR) en la Universidad Juárez Autónoma de Tabasco (UJAT), Tabasco, México; para lo cual, los peces se mantuvieron en tinas de plástico circulares de 1,7 $\mathrm{m}^{3}$ de capacidad, dentro de un sistema de recirculación abierto. Las larvas (I), fueron confinadas a una densidad de 300I/ $\mathrm{m}^{3}$, en un sistema de recirculación abierto, equipado con una bomba de 1.5hp (A.O. Smith ${ }^{\circledR}$ ) y un filtro de arena, (Series Competition ${ }^{\circledR}$ ), con tanques de 200 L. Los parámetros de la calidad del agua (temperatura, oxígeno disuelto y $\mathrm{pH}$ ) fueron medidos diariamente con un termómetro (Brannan USA), un oxímetro (YSI 55, California USA) y un potenciómetro (Denver Instrument UB-10, Denver, Colorado, USA), manteniéndose en $27.3 \pm 0.6^{\circ} \mathrm{C}$, $5.3 \pm 0.38 \mathrm{mg} / \mathrm{L}$ y $7.3 \pm 0.14$, respectivamente, durante todo el experimento.

El diseño experimental consistió de dos tratamientos, elaborados con base a una dieta experimentales, con y sin fluoximesterona (TAH y TA, respectivamente) y dos controles: positivo (SHC) y negativo (SC), con y sin hormona, respectivamente. Los alimentos $\mathrm{SCH}$ y SC fueron aplicados con base al alimento de origen comercial, EI Pedregal Silver Cup ${ }^{\circledR}$ (iniciador55\% de proteína, Nelson and Sons Inc., USA). Los tratamientos fueron realizados por triplicado. La obtención de las fuentes proteínicas no convencionales, (la sardinita de agua dulce $A$. aeneus, y el caracol tote $P$. flagellata), fue en el poblado de El Águila perteneciente al municipio de Balancán, Tabasco. Para la elaboración de la dieta experimental, las fuentes o ingredientes proteínicos no convencionales, fueron secados en un horno marca Bathammex ${ }^{\circledast}$ a $60^{\circ} \mathrm{C}$, por $2 \mathrm{~h}$ (o más, según el contenido de agua de los ingredientes). Posteriormente, se obtuvieron harinas de ambos ingredientes, empleando un molino (Nixtamatic ${ }^{\circledR}$, NTC-01, 
1/3hp), para obtener tamaños de partícula de $500 \mu \mathrm{m}$ como máximo. La dieta experimental a base de las fuentes proteínicas no convencionales, fue diseñada con el paquete de formulación de alimentos balanceados MIXIT-WIN v 5.0. Los ingredientes, para la dieta experimental, fueron pesados en una balanza marca Ohaus ${ }^{\circledR}$ (precisión $0.001 \mathrm{~g}$ ) y mezclados en una batidora de $5 \mathrm{~kg}$ (Blazer $\left.{ }^{\circledast}, \mathrm{B}-10\right)$, mezclando harinas secas y componentes húmedos (aceite de hígado de bacalao, carboximetilcelulosa y agua), por separado. Posteriormente ambos agregados (seco y húmedo) fueron mezclados de nueva cuenta en la batidora, junto con la premezcla de vitaminas, y minerales, hasta obtener una pasta semihúmeda (Gaxiola et al., 2005). Las pastas semihúmedas, fueron pasadas por un molino para carne, motor de $1 \mathrm{hp}$, monofásico (Torrey ${ }^{\oplus}, \mathrm{M} 22$ ), hasta obtener pellets de diámetro $3 \mathrm{~mm}$. Los pellets fueron secados en un horno (Bathammex ${ }^{\oplus}$ ) a $60^{\circ} \mathrm{C}$, por $8 \mathrm{~h}$ aproximadamente.

Los alimentos con hormona fluoximesterona (THA y $\mathrm{SHC}$ ), fueron preparados como describen JiménezBadillo \& Arredondo-Figueroa (2000). Primeramente, se pesó $1 \mathrm{~kg}$ de alimento previamente formulado, elaborado y molido, en un molino para café, como se describió previamente. Después de este proceso los alimentos para la obtención de THA y SHC se cribaron, en un tamiz del número 50. Dos tabletas de fluoximesterona con ingrediente activo $2,5 \mathrm{mg}$, nombre comercial Stenox ${ }^{\circledR}$, fueron trituradas en un mortero de porcelana, hasta obtener un polvo fino, el cual se disolvió en un litro de alcohol etílico absoluto (J.T. Baker ${ }^{\circledR} 99.98 \%$ de pureza), de modo que la proporción de hormona en el alimento al final, fue de $5 \mathrm{mg} / \mathrm{kg}$. Posteriormente, la solución hormonada ya homogenizada, fue rociada sobre los alimentos antes mencionados.

Un lote de 100 larvas (por réplica), fueron introducidas de manera aleatoria en las tinas de 200L. Se realizó un muestreo de larvas $(n=30)$ por réplica, para realizar una biometría inicial (y quincenales en adelante). Los alimentos con y sin hormona fueron suministrados, a crías de C. urophthalmus, al 10\% de la biomasa/día, durante 45 días de fase experimental. La frecuencia de alimentación fue de 3 veces por día. Una vez concluido el tratamiento hormonal por 45 días, los peces fueron llevados a una taIla de $7 \mathrm{~cm}$ (tres meses de edad aproximadamente), para determinar el sexo de una muestra de 30 peces por réplica. Las gónadas fueron teñidas con colorante de Wrigth y se obtuvieron los frotis, mediante la técnica del "Squash". Las preparaciones fueron observadas a 10x, 40x y 100X en el microscopio de contraste de fases (Nikon, SM800).

Para el análisis de proteína cruda de muestras de ingredientes (fuentes proteínicas no convencionales, por separado) y material peletizado de alimentos (dieta experimental y alimento comercial), se empleó la técnica de Microkjeldahl (AOAC, 1990). Las muestras fueron digeridas por separado (1g), en presencia de ácido sulfúrico concentrado (J. T. Baker ${ }^{\circledast}$ ) en tubos para digestión de $0.25 \mathrm{~L}$ (Labconco), dentro de un módulo de calentamiento de cerámica de 4 unidades (Labconco, Rapid Digestor), durante un tiempo aproximado de $1 \mathrm{~h}$, hasta que las muestras se tornaron azul-verde cristalinas (por presencia de borato de amonio), y posteriormente se siguió la destilación por $15 \mathrm{~min}$ y en presencia de $\mathrm{NaOH}$ y ácido bórico al $2 \%$, y solución indicadora de rojo de metilo-azul de metileno (Ramírez, 1993), mientras que la titulación con el borato de amonio de la muestra fue con ácido sulfúrico normalizado. Las grasas crudas fueron estimadas por diferencia de pesos (Nielsen, 2003). Las grasas se segregaron por reflujo, con éter dietílico, haciendo uso de un sistema de tubos refrigerantes.

Una vez evaporado el éter a $105^{\circ} \mathrm{C}$, se estimaron los porcentajes de inclusión de lípidos. La cuantificación de almidón siguió la técnica de Southgate, (1991), donde las muestras fueron puestas a hidrolizar en condiciones ácidas, en presencia de ácido sulfúrico $0.2 \mathrm{M}$, por $4 \mathrm{~h}$. El porcentaje de humedad fue obtenido según Nollet, (1996). Las muestras fueron secadas a $60^{\circ} \mathrm{C}$. Posteriormente las muestras fueron pesadas en seco, cuyo peso obtenido se dividió por el peso húmedo inicial y expresado en porciento.

Con lo que respecta a las pruebas estadísticas empleadas para el análisis de los datos, las tasas de crecimiento específicas, los porcentajes de masculinización y de supervivencia por tratamiento, fueron sujetos a una transformación como sugiere Zar (1974), de modo que los datos expresados en valores porcentuales, tiendan a la normalidad y homocedasticidad. Para tal fin, Cuenca et al. (2013a), sugieren la transformación de la forma:

$$
X^{\prime}=\frac{\sqrt{(X+1)}+0.375}{\sqrt{X}+15}
$$

Donde $X^{\prime}$ es el valor $\mathrm{GH}$-ésimo transformado y $X$ es el valor GH-ésimo en crudo. Por tanto, se aplicó una ANOVA de dos vías, para determinar la existencia o no, de diferencias significativas en los datos transformados. Se utilizó una prueba de Tukey, para discernir los tratamientos que difirieron entre sí. Por otro lado, para los pesos finales promedio, la ganancia en peso al día y los factores de condición al final del experimento, se aplicó la prueba no paramétrica de Friedman, para determinar si los datos resultaron significativos. Para todas las pruebas, el paquete estadístico empleado fue el Statistica TM v8. 


\section{RESULTADOS}

Los pesos iniciales promedio, de una muestra de 30 larvas de $C$. urophthalmus por réplica y tratamiento, no fueron significativos $(p>0,05)$. Los parámetros de crecimiento y sobrevivencia, por efecto de la dieta experimental y el alimento comercial (TA y SC); así como los porcentajes de masculinización, por efecto de los alimentos con hormona esteroide sintética fluoximesterona (THA y SHC), se pueden visualizar en la tabla 1. Las tasas de crecimiento de las crías de C. urophthalmus, por efecto de los distintos tratamientos, mostraron diferencias significativas entre sí $(p<0,05)$, resultando la tasa de crecimiento THA $(7.1 \pm 0.28 \%)$, significativamente mayor que las SHC, TA y SC $(6,6 \pm 0,31 ; 5,9 \pm 0,16$ y $5,0 \pm 0,98 \%$, respectivamente).

Los pesos finales promedio de igual manera, fueron significativos $(p<0,05)$, resultando el peso final promedio THA, el de mayor valor $(0,32 \pm 0,03 \mathrm{~g})$, en tanto que el tratamiento SC generó en menor peso final promedio $(0.15 \pm 0.03 \mathrm{~g})$. En la figura 1, es posible ilustrar el incremento de los pesos promedio por efecto de los distintos tratamientos, donde la curva de crecimiento de los peces bajo tratamiento THA, es la que observa una tendencia exponencial más marcada $\left(W_{f}=e^{(0.075 t-4.2981)}, R^{2}=0,9742\right)$, seguido de la tendencia de crecimiento derivado del tratamiento SHC $\left(W_{f}=e^{(0.0671 t-4.3049)}, \mathrm{R}=0,9882\right)$, donde
$W_{f}$ es el peso promedio final en gramos y $t$ el tiempo en días). La curva de crecimiento de los tratamientos TA y SC, mostraron una tendencia más linealizada. Así mismo, la longitud total promedio final y el peso ganado por día, también fueron significativos $(p<0,05)$, siendo los peces bajo efecto del tratamiento THA, los de mayor longitud y ganancia en peso por día observaron.

En lo que respecta a los valores del factor de condición en crías de $C$. urophthalmus, éstos no resultaron significativos ( $p>0,05)$. En la tabla 1 se observa que el máximo factor de condición, al cabo de 45 días de experimento, correspondió al tratamiento SC $(2,3 \pm 0,12)$, mientras que el menor factor de condición lo obtuvo el tratamiento THA $(1,74 \pm 0,11)$.

Los distintos valores del factor de conversión alimenticia, por efecto de los distintos tratamientos, se pueden apreciar en la figura 2. Los factores de conversión fueron significativos, tras 45 días de fase experimental $(p<0,05)$. Se puede visualizar que el menor factor de conversión, fue atribuible al tratamiento THA $(2,45 \pm 0,1)$, en tanto que el máximo valor de factor de conversión, correspondió al tratamiento TA $(3,02 \pm 0,05)$.

Por otro lado, los resultados estadísticos revelaron que el porcentaje de masculinización, por efecto del tratamiento THA $(52,2 \pm 2,2 \%)$, fue mayormente significativo $(p<0,05)$, que aquellos por efecto de los tratamientos TA,

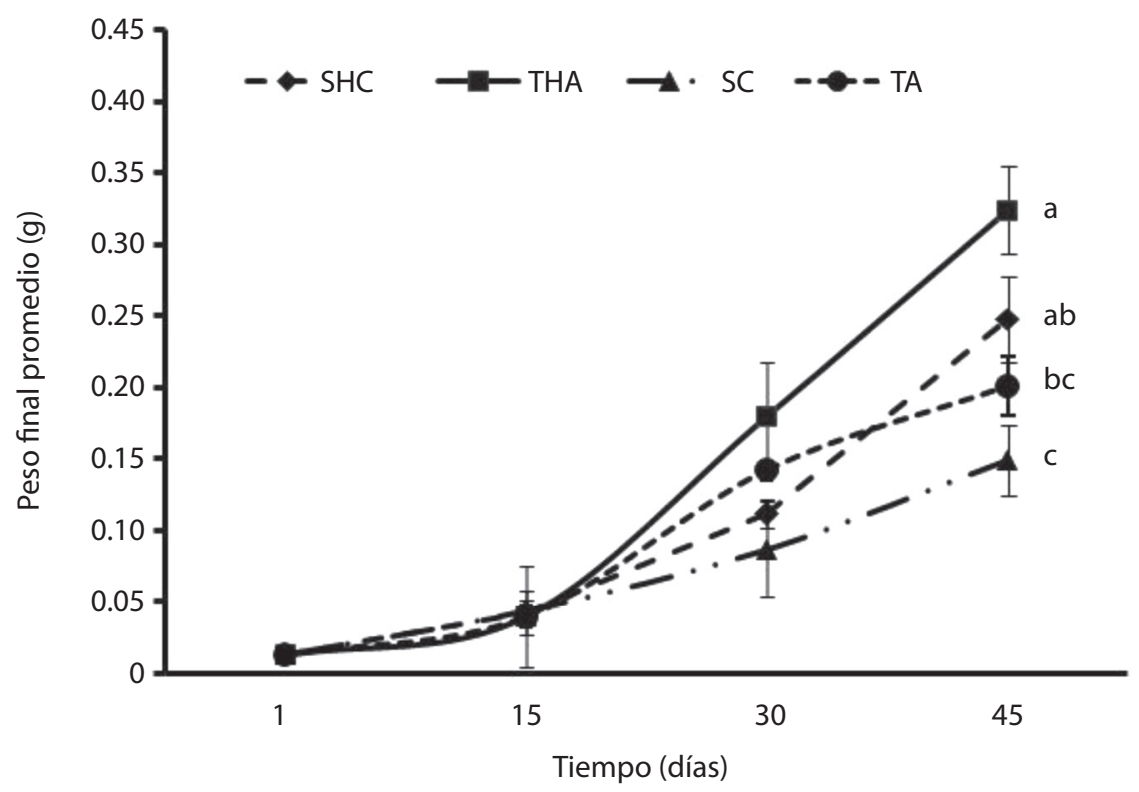

Fig. 1. Curvas de crecimiento para crías de C. urophthalmus, por efecto de los tratamientos SCH, THA, SC y TA, suministrados por 45 días. THA: Dieta experimental con fluoximesterona, TA: Dieta experimental sin fluoximesterona, SHC: Dieta a base de alimento comercial Silver Cup con fluoximesterona, SC: Dieta a base de alimento comercial Silver Cup sin fluoximesterona. Letras distintas, indican diferencias significativas. 
TABLA 1

Composición de ingredientes, composición proximal y dosis de fluoximesterona, para los tratamientos THA, TA, SHC y SC.

\begin{tabular}{|c|c|c|c|c|}
\hline Ingrediente (\%) & THA & TA & $\mathrm{SC}$ & $\mathrm{SHC}$ \\
\hline A. mexicanus & 8,0 & 8,0 & & \\
\hline P. flagellate & 82,0 & 82,0 & & \\
\hline Carboximetilcelulosa & 1,0 & 1,0 & & \\
\hline Aceite de hígado de bacalao & 2,0 & 2,0 & & \\
\hline Premezcla de vitaminas & 1,0 & 1,0 & & \\
\hline Premezcla de minerales & 1,0 & 1,0 & & \\
\hline lecitina de soya & 3,0 & 3,0 & & \\
\hline Antioxidante & 2,0 & 2,0 & & \\
\hline Composición proximal (\%) & THA & $\mathrm{TA}$ & SC & $\mathrm{SHC}$ \\
\hline Proteína cruda & $54,3 \pm 2,2$ & $54,3 \pm 2,2$ & $54,5 \pm 1,3$ & $54,5 \pm 1,3$ \\
\hline Lípidos crudos & $13,2 \pm 1,1$ & $13,2 \pm 1,1$ & $15,2 \pm 1,3$ & $15,2 \pm 1,3$ \\
\hline Humedad & $15,1 \pm 0,3$ & $15,1 \pm 0,3$ & $5,4 \pm 0,6$ & $5,4 \pm 0,6$ \\
\hline Hormona sintética (mg/kg) & THA & TA & SC & $\mathrm{SHC}$ \\
\hline Fluoximesterona & 5,0 & 0,0 & 5,0 & 0,0 \\
\hline Proporción de machos (\%) & $52.2 \pm 2.2^{\mathrm{a}}$ & $44.5 \pm 2.5^{b}$ & $44,5 \pm 2,6^{b}$ & $40,4 \pm 2,0^{b}$ \\
\hline Parámetros de crecimiento & THA & TA & SC & $\mathrm{SHC}$ \\
\hline$W_{0}(g)$ & $0,0132 \pm 0,0006^{\mathrm{a}}$ & $0,0139 \pm 0,0008^{a}$ & $0,0127 \pm 0,0009^{a}$ & $0,0142 \pm 0,0007^{a}$ \\
\hline$W_{f}(g)$ & $0,32 \pm 0,03^{a}$ & $0,20 \pm 0,02^{b c}$ & $0,15 \pm 0,03^{c}$ & $0,25 \pm 0,03^{\mathrm{ab}}$ \\
\hline $\mathrm{L}_{\mathrm{f}}(\mathrm{cm})$ & $2,65 \pm 0,08^{a}$ & $2,15 \pm 0,13^{b}$ & $1,86 \pm 0,03^{c}$ & $2,47 \pm 0,05^{a}$ \\
\hline TCE (\%) & $7,1 \pm 0,28^{\mathrm{a}}$ & $5,9 \pm 0,16^{c}$ & $5,0 \pm 0,98^{d}$ & $6,6 \pm 0,31^{\mathrm{b}}$ \\
\hline PWG (\%) & $2355,5 \pm 295,5^{a}$ & $1346,5 \pm 104,5^{c}$ & $1071,1 \pm 51,7^{d}$ & $2062,0 \pm 28,9^{b}$ \\
\hline WG/d (g) & $0,007 \pm 0,0009^{a}$ & $0,004 \pm 0,0003^{c}$ & $0,003 \pm 0,0001^{d}$ & $0,007 \pm 0,0001^{b}$ \\
\hline FCA & $2.45 \pm 0.1^{b}$ & $3,02 \pm 0,05^{\mathrm{a}}$ & $2,94 \pm 0,05^{a}$ & $2,64 \pm 0,08^{b}$ \\
\hline FC & $1,74 \pm 0,11^{\mathrm{a}}$ & $2,07 \pm 0,49^{a}$ & $2,3 \pm 0,12^{\mathrm{a}}$ & $2,04 \pm 0,03^{a}$ \\
\hline $\mathrm{S}(\%)$ & $82,7 \pm 3,1^{c}$ & $89,3 \pm 2,1^{\mathrm{ab}}$ & $91,7 \pm 2,9^{\mathrm{a}}$ & $84,3 \pm 0,6^{c}$ \\
\hline
\end{tabular}

SHC y SC $(44,5 \pm 2,5 ; 44,5 \pm 2,6$ y $40,4 \pm 2,0 \%$; respectivamente), durante 45 días de experimento.

Los porcentajes de supervivencia de cría de C. urophthalmus, por efecto de los tratamientos resultaron significativos $(p<0,05)$, siendo significativamente mayor el obtenido por el tratamiento SHC $(91,7 \pm 3,0 \%)$, mientras que el menor correspondió al tratamiento THA $(82,7 \pm 3,1 \%)$.

\section{DISCUSIÓN}

La abundancia de especies animales de alto contenido proteico, como la sardinita $A$. aeneus y el caracol tote $P$. flagellata en la región Sureste de México, fueron los criterios de peso, para su selección como ingredientes de inclusión para la dieta experimental probada en el presente estudio. Adicionalmente, son especies animales con escaso valor comercial en la región. Por otro lado, en lo que concierne al rendimiento obtenido en $C$. urophthalmus por efecto de los tratamientos probados, los peces que mayor crecimiento observaron, fueron los alimentados con las dieta experimental, a base de ingredientes animales (THA, con la inclusión de $P$. flagellata y A. aeneus en su fórmula).

Aunque el lote de crías de C. urophthalmus bajo el tratamiento THA registró la menor supervivencia y el menor factor de condición, fue el tratamiento que superó en crecimiento, al tratamiento con base al alimento comercial Silver Cup ${ }^{\circledast}$ de iniciación, con fluoximesterona. 


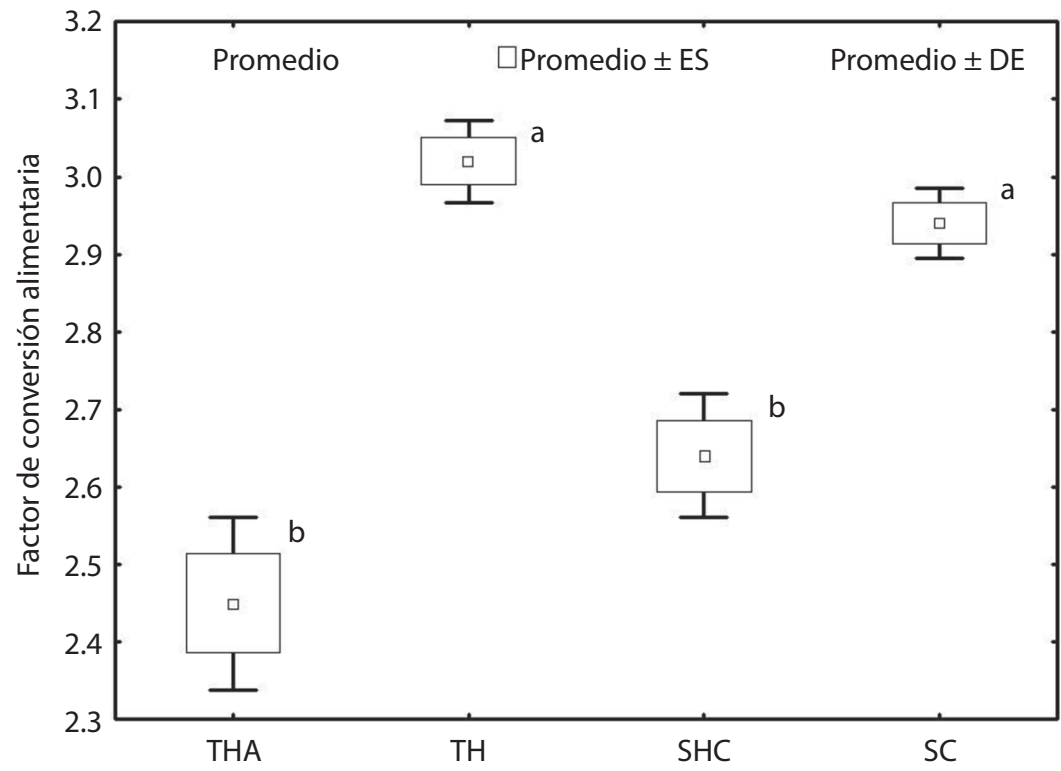

Fig. 2. Diagrama de cajas para el factor de conversión alimenticia (FCR) en crías de C. urophthalmus, por efecto de los tratamientos tras 45 días de experimento.

Martínez-Palacios \& Ross (1994), reportaron un crecimiento de crías de $C$. urophthalmus de aproximadamente $12 \mathrm{~mm}$ a los 15 días de edad, alimentadas con una dieta seca; mientras que en el presente estudio, se obtuvieron entre de 14 y $15 \mathrm{~mm}$ para el mismo período de tiempo, con la dieta TA y THA, respectivamente. JiménezBadillo \& Arredondo-Figueroa (2000) reportaron pesos finales entre 0,64 y $1,04 \mathrm{~g}$ para tres variedades de tilapia, durante su masculinización con 17-a-metiltestosterona, fluoximesterona y propionato de testosterona. Los pesos finales menores, registrados para C. urophthalmus en la presente investigación, puedan a obedecer a que en general, los peces cíclidos nativos presentan tasas de crecimiento más moderadas, con relación a tilapia.

Sin embargo, aunque no hubo diferencias significativas en los pesos finales, derivados de los tratamientos THA y SHC, las fuentes de proteínas animales no convencionales, propuestas en el presente estudio, pueden ser meritorias de investigaciones posteriores, como fuentes alternativas de proteína, con miras a diseñar alimentos inertes para C. urophthalmus, a futuro.

Por otro lado en nuestros ensayos, la tasa de crecimiento y peso promedio final de los peces tratados con el alimento THA, obtuvo aún mejores resultados, que su control negativo TA (tratamiento sin hormona). Lo anterior, posiblemente permita inferir que la fluoximesterona, pudo actuar como un agente anabólico en el tratamiento THA, debido a que fue el que generó una mayor $\mathrm{TCl}$ y peso final promedio, con respecto las crías bajo influencia del resto de lostratamientos. Con relación a esto último, Liñan-Cabello et al. (2013), revelaron un mayor incremento en la ganancia en peso por día, en crías de tilapia O. niloticus, por interacción de la hormona de crecimiento humana recombinante y la 17-a-metiltestosterona. Similares conclusiones obtuvieron Salgado et al. (2008), señalando que la hormona masculina acetato de trembolona, tuvo un efecto anabólico sobre juveniles del pez de ornato Poecilia reticulata, cuando fue tratado con alimento hormonado, a razón de $300 \mathrm{mg} / \mathrm{kg}$, por 60 días. Cabe mencionar también que en la presente investigación, un mayor rendimiento en crecimiento fue observado en el tratamiento $\mathrm{SHC}$, respecto a su control negativo, $\mathrm{SC}$.

Siguiendo con el trabajo de Liñan-Cabello et al. (2013), los autores encontraron factores de conversión alimenticia, entre 1,75 y 1,9 para crías de tilapia O. niloticus por efecto de la interacción de las hormonas antes mencionadas; mientras que en el presente estudio, los factores de conversión fluctuaron entre 2,45 y 3,02. La especie, el peso inicial, el lote genético y la edad (entre otros factores) podrían explicar tales discrepancias. La edad más temprana en peces de $C$. urophthalmus del presente experimento por ejemplo, demanda mayor cantidad de alimento, y más por tratarse de un tratamiento hormonado vía alimento. En la presente investigación no obstante, el menor factor de conversión alimenticia, fue observado en el tratamiento THA, lo que permite asumir una mejor eficiencia de conversión del alimento, que con relación al 
del resto de tratamientos (lo que incluye a los tratamientos SHC y SC, a base del alimento comercial Silver Cup).

En lo que toca a los porcentajes de masculinización, por efecto de los tratamientos THA y SHC, los resultados no arrojaron evidencia alguna de que, la hormona esteroide fluoximesterona pueda lograr poblaciones monosexo masculinas por arriba del $95 \%$, al menos no con la dosis empleada en la presente investigación ( 5 $\mathrm{mg} / \mathrm{kg}$ de alimento). Estos resultados difieren notablemente a los obtenidos por Jiménez-Badillo \& ArredondoFigueroa (2000) en condiciones de ambiente templado, haciendo uso del mismo esteroide sintético, a razón de $1 \mathrm{mg} / \mathrm{kg}$, aplicada a distintas variedades de tilapia bajo tratamiento hormonado por 30 días. Los mencionados autores reportaron un $80,6 \%$ de masculinización. Cabe mencionar que los experimentos, de los cuales se contrastan los resultados, fueron realizados en condiciones medioambientales distintas, y con diferentes especies. No obstante lo anterior, es recomendable aumentar la dosis de fluoximesterona, o incluso el porciento de la biomasa/día del alimento suministrado, en futuros experimentos. Cabe señalar que este valor (de $1 \mathrm{mg} / \mathrm{kg}$ ), fue tomado como referente, para probar la dosis de $5 \mathrm{mg} / \mathrm{kg}$ en la presente investigación.

Los parámetros de crecimiento y supervivencia de C. urophthalmus en general, mostraron un rendimiento aceptable, en las dietas a base de los ingredientes proteínicos no convencionales, $P$. flagellata y $A$. aeneus, siendo por consiguiente fuentes potenciales de proteína, como posibles elementos de inclusión nutricionales para crías de esta especie. De acuerdo con los resultados y a reserva de su limitado valor androgénico (al menos con la dosis empleada), es posible atribuir a la fluoximesterona cierto efecto anabólico, que pueda contribuir al crecimiento de C. urophthalmus.

\section{AGRADECIMIENTOS}

Agradecemos al Consejo Nacional de Ciencia y Tecnología (CONACYT-Gobierno del Estado de Tabasco, México, por el financiamiento al proyecto con clave TAB-2005-C06-16472, para realización de la presente investigación.

\section{REFERENCIAS}

A.O.A.C. (1990). Official Methods of Analysis of the Assoc. of Analysis Chemists. Eilliam Horritz, Editor.

Casas, M., Portillo, G., Aguila, N., Rodríguez, S., Sánchez, I. \& Carrillo, S. (2006). Effect of the marine algae Sargassum spp. on the productive parameters and cholesterol content of the brown shrimp. Farfantepenaeus californiensis. Rev. Biol. Mar. Oceanogr., 41(1), 97-105.

Caso-Chávez, M., Yánez-Arancibia, A. \& Lara-Domínguez, A. (1986). Biología. Ecología y dinámica de poblaciones de Cichlasoma urophthalmus (Günther) (Pisces: Cichlidae) en hábitat en Thalassia testudinum y Rhizophora mangle, Laguna de Términos, Sur del Golfo de México. Biótica, 11(2), 79-111.

Chávez-Sánchez, C., Martínez-Palacios, C.A, Martínez-Pérez, G. \& Ross, L.G. (2000). Phosphorus and calcium requirements in the diet of the American cichlid Cichlasoma urophthalmus. Aquaculture Nutrition, 6,1-9.

Chavez-Lomelí, M.O., Mattheeuws, M.H. \&Vega, P. 1989. Biología de los peces del Rio San Pedro en vista de determinar su potencial para la piscicultura. Xalapa Veracruz, México.

Cuenca C.A., Álvarez-González, C.A, Ortiz-Galindo, J.L., Guerrero-Zárate, R., Perera-García, M.A., HernándezGómez, R.E. \& Nolasco-Soria, H. (2013a). Digestibilidad in vitro de ingredientes proteínicos en la mojarra castarrica Cichlasoma urophthalmus. Ecosistemas y Recursos Agropecuarios, 29(3), 263-275.

Cuenca C.A., Álvarez-González, C.A., Ortiz-Galindo, J.L., TovarRamírez, D., Guerrero-Zárate, R., Aguilar-Hernández, S., Perera-García, M.A., Hernández-Gómez, R. \& Gisbert, E. (2013b). Histological development of the digestive system of Mayan cichlid Cichlasoma urophthalmus (Günther 1862). Journal of Applied Ichthyology. 29(6): 1304-1312.

D’artola, A.L., Arias, L. \& Páramo, S. (2008). Estudio citogenético en la sardina de agua dulce Astyanax aeneus (pisces: characidae). Semana de Divulgación y Video Científico. Villahermosa, Tabasco, México.

El-Sayed, A.F.M., Martínez, N.I \& Moyano, F.J. (2000). Assessment of the effect of plant inhibitors on digestive proteases of Nile tilapia using in vitro assays. Aquaculture International, 8, 403-415.

Frías-Quintana, C.A., Álvarez-González, C.A \& MárquezCoutoriel, G. (2010). Diseño de microdietas para el larvicultivo de pejelagarto Atractosteus tropicus (Gill 1863). Ecosistemas y Recursos Agropecuarios, 26(2), 265-282.

Gaxiola, M.G., Cuzon, T., Taboada, G., Brito, R., Chimal, M.G., Paredes, A., Soto, L., Rosas, C. \& Wormhouldt, A. (2005). Factorial effects of salinity, dietary carbohydrate and moult cycle on digestive carbohydratases and hexoquinases in Litopenaeus vannamei (Boone, 1931). Comparative Biochemistry and Physiology, 149(A), 29-39.

Iriarte-Rodríguez, F.V. \& Mendoza-Carranza, M. (2007). Validación del cultivo semiintensivo del caracol tote (Pomacea flagellata), en el trópico húmedo. Aquactic., 27, 16-30.

Jiménez-Badillo, M.L. \& Arredondo-Figueroa, J.L. (2000). Effect of oral treatment of synthetic androgens on sex ratio, survival and growth rates, in three strains of tilapia. Hidrobiológica, 10(2), 115-120. 
Lee, K.J., Dabrowski, K., Blom, J.H., Bai, S.C \& Stromberg, P.C. (2002). A mixture of cotton seed meal and animal by product mixture as a fish meal substitute: growth and tissue gossypol isomers in juvenile rainbow trout (Oncorhinchus mykiss). J. Anim. Physiol. Anim. Nutri., $86,1-13$

Liñan-Cabello M.A., C.M. Robles-Basto \& A. Mena-Herrera. 2012. Somatic growth effects of intramuscular injection of growth hormone in androgen-treated juvenile Nile tilapia, Oreochromis niloticus (Perciformes: Cichlidae). Revista de Biología Tropical, 61(1), 203-212.

Martínez-Palacios, C.A. \& Ross, L.G. (1988). The feeding ecology of the Central American cichlid Cichlasoma urophthalmus (Günther, 1862). J. of Appl. Icthyol, 8, 99-109.

Martínez-Palacios, C.A. \& Ross, L.G. (1994). Biología y cultivo de la mojarra latinoamericana Cichlasoma urophthalmus. Consejo Nacional de Ciencia y Tecnología, México.

Perera-García M. A., Mendoza-Carranza, M \& PáramoDelgadillo, S. (2008). Dinámica reproductiva y poblacional del robalo Centropomus undecimalis (perciformes: Centropomidae), en barra San Pedro, Centla, México. Ecosistemas y Recursos Agropecuarios, 24(1), 49-59.

Nielsen S. (2003). Food Analysis Laboratory Manual. Kluwer Academic/Plenum Publishers, Nueva York, USA.

Nollet, L.M.L. (1996). Handbook of food analysis. M. Dekker. Nueva York. USA.
Pérez-Sánchez, E. \& Páramo-Delgadillo, S. (2008). The culture of cichlids of the southeastern Mexico. Aquac. Res., 39, 777-783.

Ramírez de Bernal. (1993). Análisis de Alimentos. Academia Colombiana de Ciencias Exactas, Físicas y Naturales. Colección Julio Carrizosa Valenzuela. Santafé de Bogotá, Colombia.

Salgado Z.H., H.A. Azpeitia, H.S. Marañón \& P.E. Maya. 2008. Efecto anabólico y androgénico del esteroide acetato de trembolona en el guppy Poecilia reticulata. Vet. Méx, 39(3), 269-279.

Southgate, D.A.T. (1991). Determination of Food Carbohydrates. Second Edition; Elsevier Applied Science, Nueva York, USA.

Uscanga-Martínez, A., Álvarez-González, C.A., ContrerasSánchez, W.M., Márquez-Couturier, G., Civera-Cerecedo, R., Nolasco-Soria, H., Hernández-Llamas, A., GoytortúaBores, E. \& Moyano, F.G. (2012). Protein requeriment in masculinized and non-masculinized juveniles of Bay Snook Petenia splendida. Hidrobiológica, 22(3), 219-228.

Treviño, L., Álvarez-González, C.A., Perales-García, N., ArévaloGalán, L., Uscanga- Martıínez, A., Márquez-Couturier, G., Fernández, I. \& Gisbert, E. (2010). A histological study of the organogenesis of the digestive system in bay snook Petenia splendida Günther (1862), from hatching to the juvenile stage. J. Appl. Ichthiol., 27, 73-82.

Zar, J.H. (1974). Biostatistical Analysis. Prentice Hall, Englewood Clifs, New Jersey, USA. 